\title{
Enhanced spectral discrimination through the exploitation of interface effects in photon dose data
}

\author{
Paule M. Charland ${ }^{\mathrm{a})}$ and Indrin J. Chetty \\ Department of Radiation Oncology, The University of Michigan, Ann Arbor, Michigan 48109 \\ Lori D. Paniak \\ Michigan Center for Theoretical Physics, The University of Michigan, Ann Arbor, Michigan 48109 \\ Bryan P. Bednarz \\ Department of Nuclear Engineering \& Radiological Science, The University of Michigan, Ann Arbor, \\ Michigan 48109 \\ Benedick A. Fraass \\ Department of Radiation Oncology, The University of Michigan, Ann Arbor, Michigan 48109
}

(Received 25 November 2002; revised 18 July 2003; accepted for publication 7 November 2003; published 23 January 2004)

The convolution/superposition algorithm for computing dose from photon beams in radiation therapy planning requires knowledge of the energy spectrum. The algorithm can compute the dose for a polyenergetic beam as the weighted sum of the individual dose contributions from monoenergetic beams. In this study we exploit interface effects apparent in the dose distributions to discriminate among spectra of high energy photon beams. We have studied the sensitivity of the depth dose distribution to the energy components using a hypothetical beam for various field sizes and depths in water and water-lung-water media. Six theoretical spectra were simulated. We compared depth dose data from these spectra using three quantitative measures which are inherently free of normalization ambiguities: for homogeneous water, the ratio $D_{20} / D_{10}$ and a logarithmic derivative in the buildup region $\mathrm{LD}_{\text {build-up }}$ and for inhomogeneous lung/water, the lung correction factor (CF). It was found that the ability of both the $\mathrm{CF}$ and the $\mathrm{LD}_{\text {build-up }}$ tests to discriminate between the various theoretical spectra were superior to that of the $D_{20} / D_{10}$ test. This discriminating power of the $\mathrm{CF}$ test decreases with increasing field size due to restored electronic equilibrium. The $\mathrm{CF}$ test, though, has some advantages over the $\mathrm{LD}_{\text {build-up }}$ test since it is less prone to electron contamination issues and numerical errors. A practical example with a $15 \mathrm{MV}$ photon beam illustrates the process. Consequently, we suggest that as part of a beam-commissioning methodology, designated electronic disequilibrium test cases be implemented in unambiguously determining the correct energy spectrum to be used. () 2004 American Association of Physicists in Medicine. [DOI: $10.1118 / 1.1637731]$

Key words: photon spectrum, convolution, lung, dose calculation, build-up, normalization

\section{INTRODUCTION}

Modern dose calculation algorithms for treatment planning attempt to better account for the details of the radiation transport. One major implementation difference between the newer algorithms and the previous semiempirical type of algorithms is explicit energy dependence. An example of an algorithm requiring the spectrum of a linac as input is the convolution/superposition ${ }^{1-4}$ method. It is not a simple task to unambiguously identify the energy spectrum producing a given dose distribution. Even starting with a spectrum obtained through one of several methods, typically there is fine tuning of the spectrum to match the measured dose data. ${ }^{5}$ This is because all methods to solve for the spectrum, whether they are direct or indirect, have limitations. First, direct measurements are impractical and difficult. ${ }^{6-8}$ Also, the inverse radiation transport problem, whether it is based on transmission measurements, ${ }^{9-14}$ transmission with build-up measurements, ${ }^{15}$ or linear combination of monoenergetic data, ${ }^{16-18}$ is ill-conditioned and any approach can at best yield an approximate solution. Moreover, the Monte Carlo simulation of the treatment head of a linac to generate photon beams is sensitive to the input parameters such as the various machine specifications. ${ }^{19-23}$ Hence, one ends up relying on a trial-and-error approach to find a spectrum that will yield the measured dose data.

This process of fitting a spectrum normally uses dose data measured in homogeneous water. ${ }^{5}$ Commonly, works on spectral determination, in addition to limiting their process to homogeneous water data, ${ }^{5,24}$ show few fits to measured dose data, or even a single comparison to a tissue-maximum-ratio or TMR for a single field size, ${ }^{9}$ for the purpose of their presentation. Though very informative about their methodology, it is not possible to ascertain how appropriate their spectrum is in more complicated irradiation situations. But, in fact we will see in a simulation that for the common situation of a water-equivalent medium, there are a number of energy spectra producing essentially the same dose via the convolution/superposition algorithm. The quantitative determination of equivalent dose in this case is assessed via the 
normalization-independent $D_{20} / D_{10}$ test. ${ }^{25}$ Since the $D_{20} / D_{10}$ test is not a very good discriminator of spectra, our objective is to introduce new tests for quantitatively distinguishing energy spectra through the examination of associated depth dose data.

We demonstrate that the physics of radiation in an inhomogeneous medium provides information that can be used to enhance spectral discrimination. Using a lung correction factor (CF)-type test, we are able to quantify our results in inhomogeneous media. This test shares normalization independence with $D_{20} / D_{10}$. The validation of any dose calculation algorithm requires a comprehensive set of test cases. ${ }^{26}$ With the use of convolution/superposition as a powerful mathematical method for dose calculation, appropriate testing needs to be elaborated and criteria allowed to evolve. Such a testing effort has been recently presented with the use of a variety of irradiation conditions and the inclusion of inhomogeneous media to verify the accuracy of the convolution/ superposition method. ${ }^{27}$ The inhomogeneous phantoms in this case were intended as a postvalidation of the algorithm rather than a test for spectrum discrimination. In this study we will propose a methodology for discriminating a spectrum based on the physics of radiation in inhomogeneous media.

In addition to the $D_{20} / D_{10}$ and CF tests, we will also investigate the possibility of using data from the build-up region of a dose curve in a water homogeneous medium in order to discriminate spectra producing these doses. In order to access the information contained in the build-up region, we have devised the $\mathrm{LD}_{\text {build-up }}$ test which involves a logarithmic derivative of the depth dose curve. The usefulness of such a test is suggested by the philosophy behind the CF test: we can obtain physical information about spectra from the doses they produce following an interface such as water/lung or air/water. The $\mathrm{LD}_{\text {build-up }}$ test shares in common with the $D_{20} / D_{10}$ and CF tests the absence of normalization dependence. A practical example for a $15 \mathrm{MV}$ photon beam making use of the CF test is included to illustrate the potential application of the process.

\section{METHODS}

In order to demonstrate the tests for resolving spectra, hypothetical dose data have been calculated via the convolution/superposition algorithm. The algorithm was further used for a practical example.

\section{A. Dose calculation algorithm}

There exist various implementations of the convolution/ superposition algorithm for a polyenergetic spectrum. In the component implementation used in this paper (Appendix A), the energy dependence is left explicit as opposed to preaveraged. That is, the dose is computed as a fluence-weighted sum of monoenergetic beams. This allows appropriate modeling of beam hardening. It also makes iterative spectrum fitting easier. The algorithm has been written in FORTRAN, and implemented inside the in-house UMPlan 3D treatment planning system. The algorithm is a modified version of
Mackie's original convolution algorithm, ${ }^{1}$ allows fine grid dose calculations, and includes various implementation features. The energy deposition kernels (EDK) used in this work are tilted and were Monte Carlo generated. ${ }^{1}$ The kernels are density scaled in regions of inhomogeneity during superposition. In reality, the spectrum varies off-axis. In our study, a single central axis spectrum with only an off-axis softening correction was used. In some implementations, it is possible to have different spectra to improve the fit to measured data over a large range of field sizes and wedges. ${ }^{5,24}$ The electron contamination term contained within the UMPlan version of the algorithm was turned off for this investigation. "Electron contamination" throughout the paper refers also to low energy photons present in the main spectrum resulting from contaminant electrons.

The calculation of the component convolution/ superposition algorithm for an arbitrary energy spectrum is prohibitively expensive in calculation time. Since calculations are always performed via numerical methods on computers, we always deal with a discretized version of the energy spectrum represented by binned data. In order to obtain good results, one requires a large number of discrete bins to accurately model the energy spectrum and, because the convolution/superposition algorithm is a linear process, the calculation time increases linearly with the number of bins. Given current computing power, the time needed to obtain satisfactory results for the dose distribution is impractical for planning optimization. ${ }^{28}$ In order to improve computational performance of the convolution/superposition algorithm, we have arbitrarily chosen to replace the physical spectrum by a small number of components. This replacement, which leads to great economy of calculation, also produces clinically acceptable results for dose across a wide range of common field sizes. We expect our results to hold for multiple components for a reason which we will expand on in Sec. IV. The proposed methods are also applicable to the polyenergetic approximation of the code (Appendix A). A single set of data generated with the polyenergetic approximation of the code was used for illustration and comparison.

\section{B. Simulation method}

Our objective is twofold. First, we would like to demonstrate that the doses produced by several different sets of discrete spectra in the convolution algorithm are essentially degenerate from the point of view of the $D_{20} / D_{10}$ test. Then, we will demonstrate that the $\mathrm{CF}$ and $\mathrm{LD}_{\text {build-up }}$ tests help to break this degeneracy. We proceed to do this via a simulation with six different sets of discrete spectra.

\section{Test spectra}

The simulation used hypothetical beams generated by varying the weights of three fixed energy bins. We have selected: $0.5,2.0$, and $10 \mathrm{MeV}$ as representative energy bins. The weights of these energies have alternatively been assigned the values $0.1,0.3$, and 0.6 , so that six different spectra were generated with the weights normalized to unity. Both homogeneous water and water-lung media served as a platform for the testing. The water-lung media consisted of 
water with $6 \mathrm{~cm}$ lung of 0.3 density relative to water inserted at a depth of $4 \mathrm{~cm}$. The study was completed for 3 $\times 3 \mathrm{~cm}^{2}, 5 \times 5 \mathrm{~cm}^{2}, 10 \times 10 \mathrm{~cm}^{2}$, and $20 \times 20 \mathrm{~cm}^{2}$ beams with $90 \mathrm{~cm}$ source-surface distance (SSD). Central axis depth doses were calculated for each theoretical spectrum and phantom.

For a single field size, i.e., $3 \times 3 \mathrm{~cm}^{2}$, the simulation was repeated with the polyenergetic implementation of the convolution/superposition algorithm. The aforementioned six spectra were used as input for both the terma calculation and for the fluence-weighted preaveraging of the kernels. To account for the hardening of the beam, ${ }^{29}$ three kernels were used per beam. The kernels were calculated at depths of 0 , 10 , and $20 \mathrm{~cm}$ in water.

\section{Dose normalization in water}

The $D_{20} / D_{10}$ ratios, defined as the ratio of the dose at depth of $20 \mathrm{~cm}$ to the dose at $10 \mathrm{~cm}$, have been calculated for the water depth doses of each of the theoretical spectra. The $D_{20} / D_{10}{ }^{25}$ ratio is an alternative to the $\mathrm{TPR}_{20} / \mathrm{TPR}_{10}{ }^{30}$ ratio. The $D_{20} / D_{10}$ ratio was one of the parameters used by Lydon $^{24}$ for the commissioning of a commercial convolution algorithm, and we will simply refer to it as the $D_{20} / D_{10}$ test.

The depth dose curves in water have also been normalized to their area under the curve (NA). The depth dose curves were imported into the computational package MATHEMATICA (Wolfram Research Inc., Champaign, IL). An interpolation function was generated for each of these curves. The tail of the depth dose for depths beyond $30 \mathrm{~cm}$ was fit to an exponential of the form $A^{*} \exp \left(-B^{*}\right.$ depth $)$. Such a fitting function is believed to be a good choice representative of the real physical situation. Depth doses can be fitted to a sum of exponentials, one of which is expected to be dominant at greater depths. To do the exponential fit, data from 26 to 42 $\mathrm{cm}$ depth were used. An overlap of the fit with the data gave us confidence in the fit beyond $30 \mathrm{~cm}$ depth. The depth dose curves extrapolated to infinity with an exponential tail were integrated up to infinity. With this normalization, the observation of the dose in the build-up region (up to the maximum dose) will be referred to as the build-up test, $\mathrm{NA}_{\text {build-up }}$.

In addition, a different approach was used to examine the depth doses in water that allowed us to look more closely at the build-up region. We have taken the logarithmic derivative with respect to depth $(x)$ of the depth doses in water $D(x)$. We will refer to the result as the quantity $\mathrm{LD}_{\text {build-up }}$ as follows:

$$
\mathrm{LD}_{\text {build-up }}=\frac{d \ln D(x)}{d x}=\frac{D^{\prime}(x)}{D(x)} .
$$

Clearly, if $D(x)$ is rescaled by a constant, it would be of no consequence to the logarithmic derivative. Consequently, normalization has no effect on this test. The calculations were also executed within the MATHEMATICA software from the depth dose data treated as an interpolated function.



FIG. 1. The lung correction factor or CF is defined as the ratio of the dose in the lung phantom to the dose in the homogeneous water phantom at the same physical depth and for the same irradiation conditions.

\section{Dose normalization in inhomogeneous media}

Due to the nature of the CF test, we will not require any explicit normalization of the doses produced in inhomogeneous media. The lung correction factors $\mathrm{CF}$ have been calculated from the depth doses of each given spectrum (CF test). The CF as calculated for a given spectrum is defined as the ratio of the dose in the inhomogeneous lung phantom to the dose in the homogeneous water phantom at the same physical depth and for the same irradiation conditions (Fig. 1). We consider the independence of the CF test from any normalization prescription to be a strength which protects the test from the introduction of any unintended bias and simplifies its calculation as well. One notable feature of the CF test is that it is insensitive to electron contamination. Details of this claim can be found in Appendix B.

\section{Spectral resolution}

In order to determine the ability of each of the tests to uniquely identify a spectrum, we need to introduce a measure of "distance" between spectra for each test. In this manner, each test reduces the comparison of two depth doses generated by distinct spectra to a single number. From these data we will be in a position to judge the relative resolving ability of the different tests.

The result of a $D_{20} / D_{10}$ test is a single value, and the separation $S_{D 20 / D 10}$ between test data from spectrum $i$ and $j$ is given by the following:

$$
S_{D_{20} / D_{10}}(i, j)=\left|\frac{D_{20} / D_{10}(i)-D_{20} / D_{10}(j)}{D_{20} / D_{10}(i)+D_{20} / D_{10}(j)}\right| \times 200 \% .
$$

For the build-up test $\mathrm{LD}_{\text {build-up }}$, the separation between two spectra was found by considering points between the surface and the maximum dose (between 0.75 and $2 \mathrm{~cm}$ ). From these points we chose the separation $S_{\mathrm{LD}}$ to be the maximum value as follows: 


$$
S_{\mathrm{LD}}(i, j)=\operatorname{Max}_{x}\left|\frac{\mathrm{LD}_{i}(x)-\mathrm{LD}_{j}(x)}{\mathrm{LD}_{i}(x)+\mathrm{LD}_{j}(x)}\right| \times 200 \% .
$$

Similarly, the full region within the lung was considered to determine the resolution of the $\mathrm{CF}$ test

$$
S_{\mathrm{CF}}(i, j)=\operatorname{Max}_{x}\left|\frac{\mathrm{CF}_{i}(x)-\mathrm{CF}_{j}(x)}{\mathrm{CF}_{i}(x)+\mathrm{CF}_{j}(x)}\right| \times 200 \% .
$$

For the build-up test normalized with respect to total dose, we analogously define $S_{\mathrm{NA}}$.

The robustness of two tests was compared by taking the ratio of the relevant separations $S$.

\section{Experimental method}

An example of the application of the spectrum fitting methodology suggested in this paper is included. The intent is to define a spectrum for the convolution/superposition algorithm which will reproduce the data from a $15 \mathrm{MV}$ photon beam from a Varian Clinac 21-EX (Varian Associates, Palo Alto, CA). Measurements included depth doses in homogeneous water and inhomogeneous lung phantoms for field sizes defined at $100 \mathrm{~cm}$ from the source ranging from 3 $\times 3 \mathrm{~cm}^{2}$ up to $20 \times 20 \mathrm{~cm}^{2}$.

\section{Measurements of beam data}

The phantoms used for this investigation included homogeneous solid water slabs of density $1.015 \mathrm{~g} / \mathrm{cm}^{3}$ (Gammex RMI, Middleton WI) and inhomogeneous water-lung equivalent slab phantoms. The total phantom size was at least $30 \mathrm{~cm}$ square (ranging up to $40 \times 40 \mathrm{~cm}$ for some slabs) by $30 \mathrm{~cm}$ thick. For the full slab inhomogeneous phantom, the phantom material from depths of 4 to $10 \mathrm{~cm}$ was replaced with a $6 \mathrm{~cm}$ thick lung-equivalent full slab phantom of density $0.300 \mathrm{~g} / \mathrm{cm}^{3}$ (Gammex RMI, Middleton WI). These measurements allowed the calculation of the lung correction factors CF. The Scanditronix-Wellhöfer water phantom system (Scanditronix-Wellhöfer, Uppsala, Sweden) was also used for the ionization measurements of depth doses in homogeneous water. The IC-10 (Wellhofer Dosimetrie, Germany) ionization chamber with an outer and inner diameter of 6.8 and $6.0 \mathrm{~mm}$, respectively (wall thickness of $0.4 \mathrm{~mm}$ and effective density of $1.76 \mathrm{~g} / \mathrm{cm}^{3}$ ), was used. This corresponds to a wall of $70 \mathrm{mg} / \mathrm{cm}^{2}$ for the IC-10, which is comparable to the Farmer-type chamber $\left(65 \mathrm{mg} / \mathrm{cm}^{2}\right)$ used by Rice et $_{\text {al. }}{ }^{31}$ The charge was collected with a PRM model SH-1 (Precision Radiation Measurements, Tennessee) electrometer operated at 300 volts. The IC-10 was inserted into the phantom along the central axis of the beam at depths ranging from 1 to $20 \mathrm{~cm}$ in solid water in order to generate depth doses. The chamber was aligned with the field crosshair lines. The effective point of measurement ${ }^{30}$ of the chamber was taken into consideration for the depth positioning (upstream by $1.8 \mathrm{~mm}$ ). All measurements were carried out with a fixed source-surface distance $(\mathrm{SSD})$ of $90 \mathrm{~cm}$. The uncertainties for the IC-10 chamber, based on the reproducibility of readings repeated up to three times, were less than $1 \%$. The measurement sessions lasted a few hours. Some of the readings taken at the beginning of the session were re- peated at the end to estimate any possible drift in output of the linac or chamber sensitivity. These differences were less than $1 \%$.

\section{Spectrum fitting procedure}

The physical 15 MV spectrum data have been modeled for the convolution/superposition algorithm using the criteria of the CF values for a $3 \times 3 \mathrm{~cm}^{2}$ field. Two energy bins were selected as a basis for the modeled convolution spectrum. The experimental lung correction factor $\mathrm{CF}_{\exp }$ had to be matched to the modeled one, $\mathrm{CF}_{\text {mod }}$ :

$$
\mathrm{CF}_{\text {mod }}=\frac{w_{1} \cdot D_{1}^{\text {lung }}+w_{2} \cdot D_{2}^{\text {lung }}}{w_{1} \cdot D_{1}^{\text {water }}+w_{2} \cdot D_{2}^{\text {water }}} \text {. }
$$

The weights of the spectral components of energy $E_{1}$ and $E_{2}$ are represented by $w_{1}$ and $w_{2}$, respectively. The dose in the lung inhomogeneous phantom is labeled as $D_{i}^{\text {lung }}$ for an energy component $i$ and $D_{i}^{\text {water }}$ is the corresponding dose in the homogeneous water. The numerator and denominator of the previous equation are divided by $w_{2}$ and the ratio of the weight $w_{1} / w_{2}$ is set to $\lambda$

$$
\mathrm{CF}_{\text {mod }}=\frac{\lambda \cdot D_{1}^{\text {lung }}+D_{2}^{\text {lung }}}{\lambda \cdot D_{1}^{\text {water }}+D_{2}^{\text {water }}} .
$$

For the fitting procedure we used a constrained least-squares minimization algorithm from the MATHEMATICA software. Our objective is to find $\lambda$, constrained to be positive such that the difference between $\mathrm{CF}_{\text {exp }}$ and $\mathrm{CF}_{\text {mod }}$ is minimized

$$
\operatorname{Min}_{x}\left|\mathrm{CF}_{\text {exp }}(x)-\mathrm{CF}_{\text {mod }}(x)\right|, \quad \lambda>0 .
$$

The energy components $E_{1}$ and $E_{2}$ are chosen by trial and error. While we have no definite method for choosing $E_{1}$ and $E_{2}$, the observation of the CF curves for a number of different energies offers some intuition in the selection of the components. One of the components is chosen from the "low" energy regimen for which little to no dose reduction is observed inside the lung and a higher energy component for which the dose drops considerably inside the lung slab due to electronic disequilibrium. Equations (5)-(7) can be generalized to include more energy components.

\section{RESULTS}

\section{A. Simulation}

\section{Identifying spectra in water}

Figure 2 shows an example of simulated depth doses in water for each of the theoretical spectra for a $3 \times 3 \mathrm{~cm}^{2}$ field. The normalizations of these curves at this point are left arbitrary. We have included units but the magnitude is arbitrary. In spite of this ambiguity, we are still able to extract useful information. For example, we can calculate the $D_{20} / D_{10}$ values and depths of maximum dose, $d_{\max }$, for these depth doses. The values are presented in Table I. As can be seen in this table, the $D_{20} / D_{10}$ values are clustered pairwise when the high-energy component $(10 \mathrm{MeV})$ of the two spectra are weighed the same. Jeraj and his coauthors ${ }^{32}$ also observed that depth doses in water were similar when the high-energy 


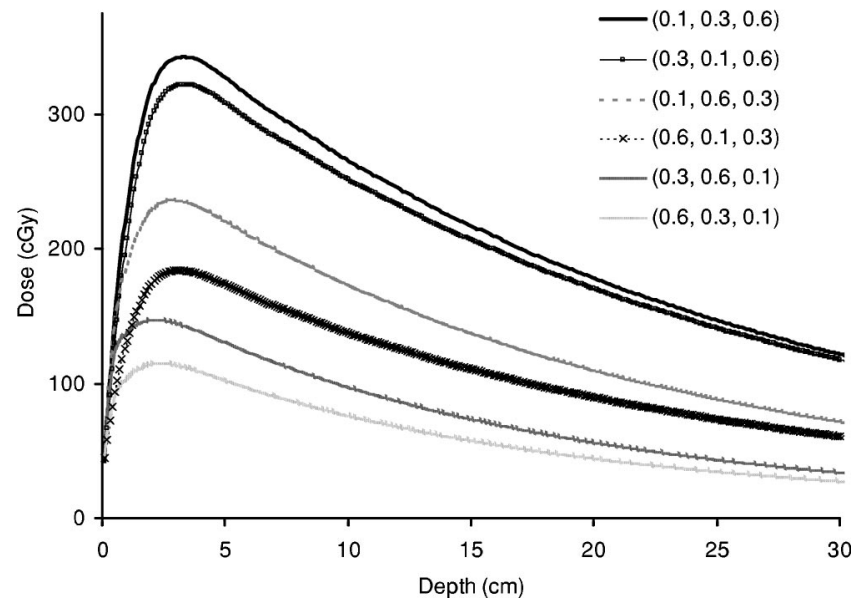

FIG. 2. Depth dose comparison in homogeneous water between different spectra for a field size of $3 \times 3 \mathrm{~cm}^{2}$. Depth dose curves are not normalized. The component method of the convolution algorithm is employed. The weights of the $0.5,2$, and $10 \mathrm{MeV}$ energy bins, respectively, appear in the legend.

part of a spectrum was similar. Figure 3 shows the depth doses in the polyenergetic approximation compared to the corresponding ones generated with the component implementation for the same spectra and $3 \times 3 \mathrm{~cm}^{2}$ field. The depth doses in the polyenergetic approximation are normalized so that the dose at depth of $10 \mathrm{~cm}$ is the same as in the depth dose of the corresponding spectra in the component method. The polyenergetic approximation yields depth dose curves that differ from the ones from the component method in both absolute and relative dose terms, i.e., more than $10 \%$ difference for some spectra.

Similarly, the $\mathrm{LD}_{\text {build-up }}$ test can be done in water using the data from Fig. 2 but requires more analysis, including the calculation of numerical derivatives. The results of this test are shown in Fig. 4. Evident in this figure is the noise associated with the numerical analysis. Even with the noise, several important features are clearly evident. First, a dispersion of the curves is observed below a depth of about $2 \mathrm{~cm}$. A closer look shows that the curves are clustered pairwise as noted before in the $D_{20} / D_{10}$ values, but still distinct. At depths beyond the build-up, all the curves appear indistinguishable and approach a constant negative value. Recall that

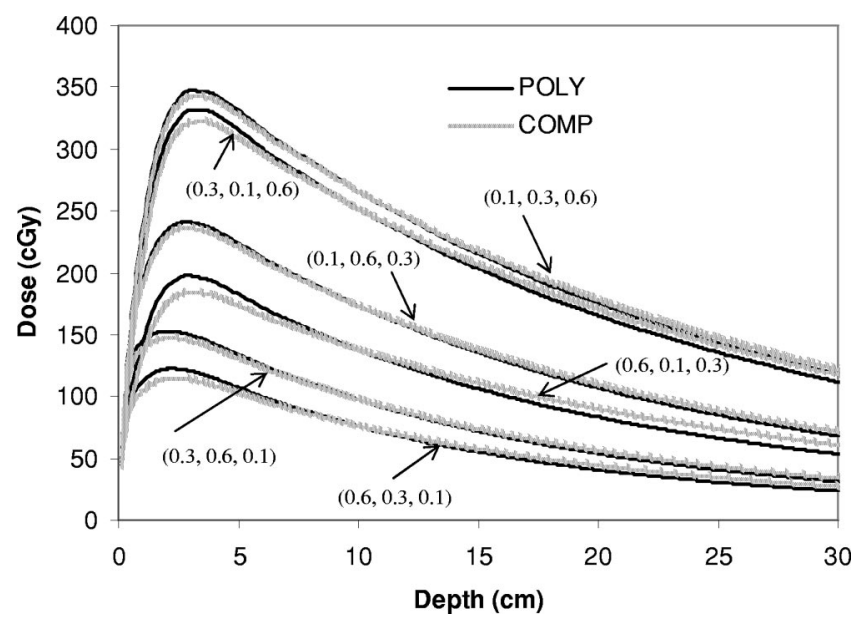

FIG. 3. Depth dose comparison in homogeneous water between the component method and the polyenergetic approximation of the convolution/ superposition code. The depth doses for the same six different spectra as in Fig. 1 for a field size of $3 \times 3 \mathrm{~cm}^{2}$ are illustrated. Depth dose curves for the polyenergetic approximation have their normalization forced to the same dose at depth of $10 \mathrm{~cm}$ as in the depth dose curves of the corresponding spectra in the component version of the algorithm.

the tail of the depth dose curves fits an exponential form. The constant value corresponds then to the attenuation coefficient.

In Fig. 5 the depth doses from Fig. 2 have been normalized with respect to their respective areas under the curvethe total doses. We would like to draw attention to several features in this figure. The intersections of different curves occur at various depths beyond $10 \mathrm{~cm}$. In addition, all curves are well distinguishable from one another around the maximum doses with the now-familiar pairwise clustering.

\section{Identifying spectra in inhomogeneous media}

Figure 6 shows depth doses in the lung inhomogeneous phantom for each of the theoretical spectrum for a 3 $\times 3 \mathrm{~cm}^{2}$ field. Normalizations are arbitrary but are the same as those for respective spectra in water (Fig. 2). Cursory observation of the plots reveals a dose reduction inside the lung slab which is located between 4 and $10 \mathrm{~cm}$ depths.

We will use the ratio of respective dose curves from Fig. 6 and Fig. 2 in order to produce the lung correction factors CF (Fig. 7). In this way we created a normalization-free measure of beam quality. In the proximal area to the lung slab,

TABLE I. $D_{20} / D_{10}$ values and depths of maximum dose for each of the six spectra for all field sizes. The first column contains the weights for the following energy components: $0.5,2$, and $10 \mathrm{MeV}$, respectively.

\begin{tabular}{|c|c|c|c|c|c|c|c|c|c|}
\hline & \multirow[b]{2}{*}{ Spectrum } & \multicolumn{2}{|c|}{$3 \times 3 \mathrm{~cm}^{2}$} & \multicolumn{2}{|c|}{$5 \times 5 \mathrm{~cm}^{2}$} & \multicolumn{2}{|c|}{$10 \times 10 \mathrm{~cm}^{2}$} & \multicolumn{2}{|c|}{$20 \times 20 \mathrm{~cm}^{2}$} \\
\hline & & $D_{20} / D_{10}$ & $\begin{array}{l}d_{\max } \\
(\mathrm{cm})\end{array}$ & $D_{20} / D_{10}$ & $\begin{array}{l}d_{\max } \\
(\mathrm{cm})\end{array}$ & $D_{20} / D_{10}$ & $\begin{array}{l}d_{\max } \\
(\mathrm{cm})\end{array}$ & $D_{20} / D_{10}$ & $\begin{array}{l}d_{\max } \\
(\mathrm{cm})\end{array}$ \\
\hline 1 & $0.1,0.3,0.6$ & 0.672 & 3.3 & 0.662 & 3.7 & 0.666 & 3.7 & 0.690 & 3.7 \\
\hline 2 & $0.3,0.1,0.6$ & 0.678 & 3.5 & 0.667 & 3.7 & 0.670 & 3.7 & 0.692 & 3.9 \\
\hline 3 & $0.1,0.6,0.3$ & 0.634 & 2.9 & 0.630 & 3.5 & 0.636 & 3.7 & 0.665 & 3.5 \\
\hline 4 & $0.6,0.1,0.3$ & 0.654 & 3.1 & 0.645 & 3.7 & 0.647 & 3.7 & 0.668 & 3.7 \\
\hline 5 & $0.3,0.6,0.1$ & 0.576 & 2.2 & 0.579 & 2.7 & 0.587 & 2.9 & 0.622 & 3.1 \\
\hline 6 & $0.6,0.3,0.1$ & 0.582 & 2.3 & 0.582 & 2.9 & 0.586 & 3.1 & 0.614 & 3.3 \\
\hline
\end{tabular}






FIG. 4. Comparison of $\mathrm{LD}_{\text {build-up }}$ for the different spectra for a field size of $3 \times 3 \mathrm{~cm}^{2}$. The component method of the convolution algorithm is employed. The logarithmic derivative is shown up to a depth of $5 \mathrm{~cm}$ since beyond this depth the curves in water remain flat.

the CF values are all near unity since no effect is expected from the distant lung slab. Therefore, the dose should be the same as in homogeneous water. The CF value drops inside the lung slab due to lack of photon scatter and increased range of the electron in the lower density region. At depths beyond the lung, the $\mathrm{CF}$ values are higher than unity due to increased penetrability of the beam in the inhomogeneous phantom as compared to the homogeneous situation due to the lack of attenuation of the primary beam inside the lung slab. The CF values also appear constant on the distal side well deep in the phantom. That is, the depth dose at depths beyond a lung slab in an inhomogeneous phantom is higher and parallel to the corresponding homogeneous case.

All the CF curves are indistinguishable at shallow depths, prior to the lung slab. Beyond the lung slab, the CF curves are clustered pairwise and barely distinguishable from one another. Within the lung region, dispersion among all the CF curves is achieved.

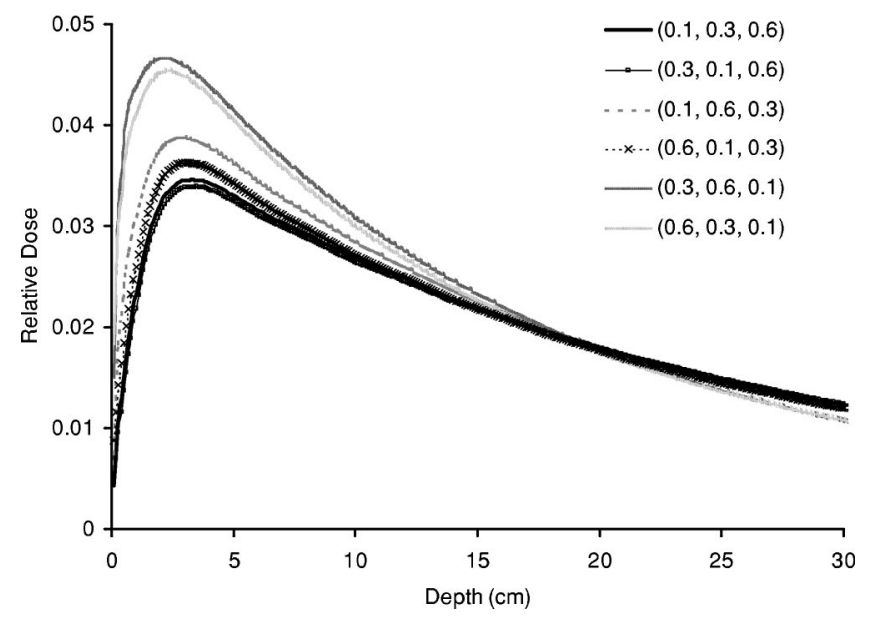

FIG. 5. Depth dose comparison in homogeneous water between different spectra for a field size of $3 \times 3 \mathrm{~cm}^{2}$. Depth dose curves are normalized to their area under the curve. The component method of the convolution algorithm is employed. The weights of the $0.5,2$, and $10 \mathrm{MeV}$ energy bins, respectively, appear in the legend.

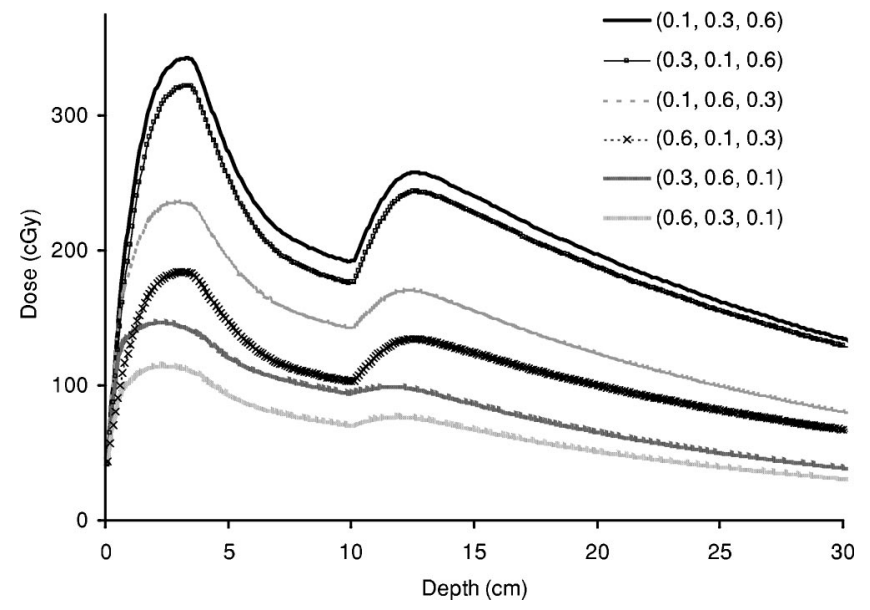

FIG. 6. Depth dose comparison in inhomogeneous water/lung phantom between different spectra for a field size of $3 \times 3 \mathrm{~cm}^{2}$. Depth dose curves are not normalized. The component method of the convolution algorithm is employed. The weights of the $0.5,2$, and $10 \mathrm{MeV}$ energy bins, respectively, appear in the legend.

The CF curves for the polyenergetic approximation of the convolution/superposition algorithm have been generated for the six theoretical spectra. Figure 8 shows the resulting curves superimposed with the corresponding ones from the component method. Dispersion among the spectra occurs inside the lung slab for the polyenergetic implementation. The polyenergetic approximation does not yield, however, the same CF curves as for the component method. This is not surprising when one considers that even in water the depth doses did not match perfectly (Fig. 3).

\section{Comparison of test-resolving power}

Given two dose curves associated with two different spectra $(i, j)$, we have different tests that give measures of the separation $S(i, j)$ of these two curves. Each test will provide a different value for the separation. Tests that yield a greater

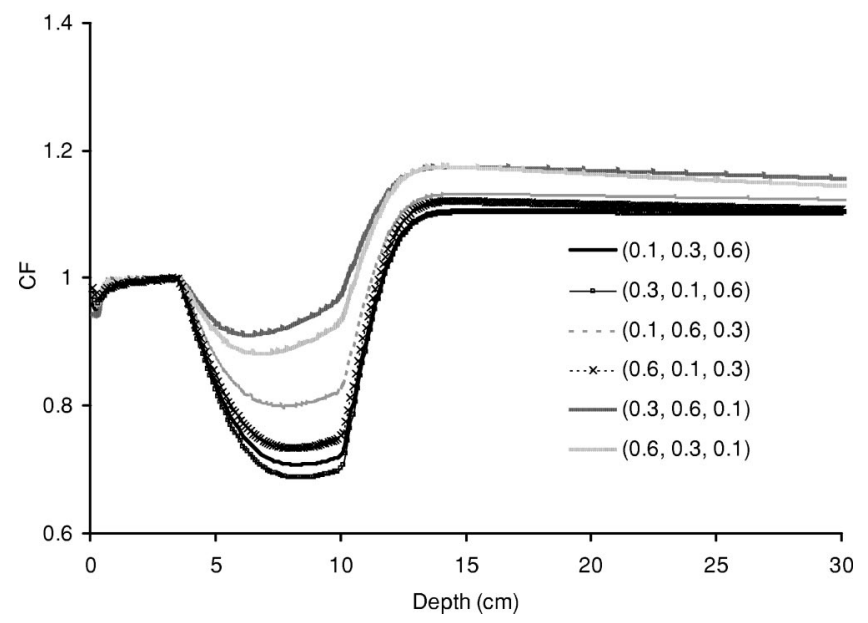

FIG. 7. The lung correction factors or CF curves for the six different spectra are shown for a field size of $3 \times 3 \mathrm{~cm}^{2}$. The component method of the convolution algorithm is employed. The weights of the $0.5,2$, and $10 \mathrm{MeV}$ energy bins, respectively, appear in the legend. 

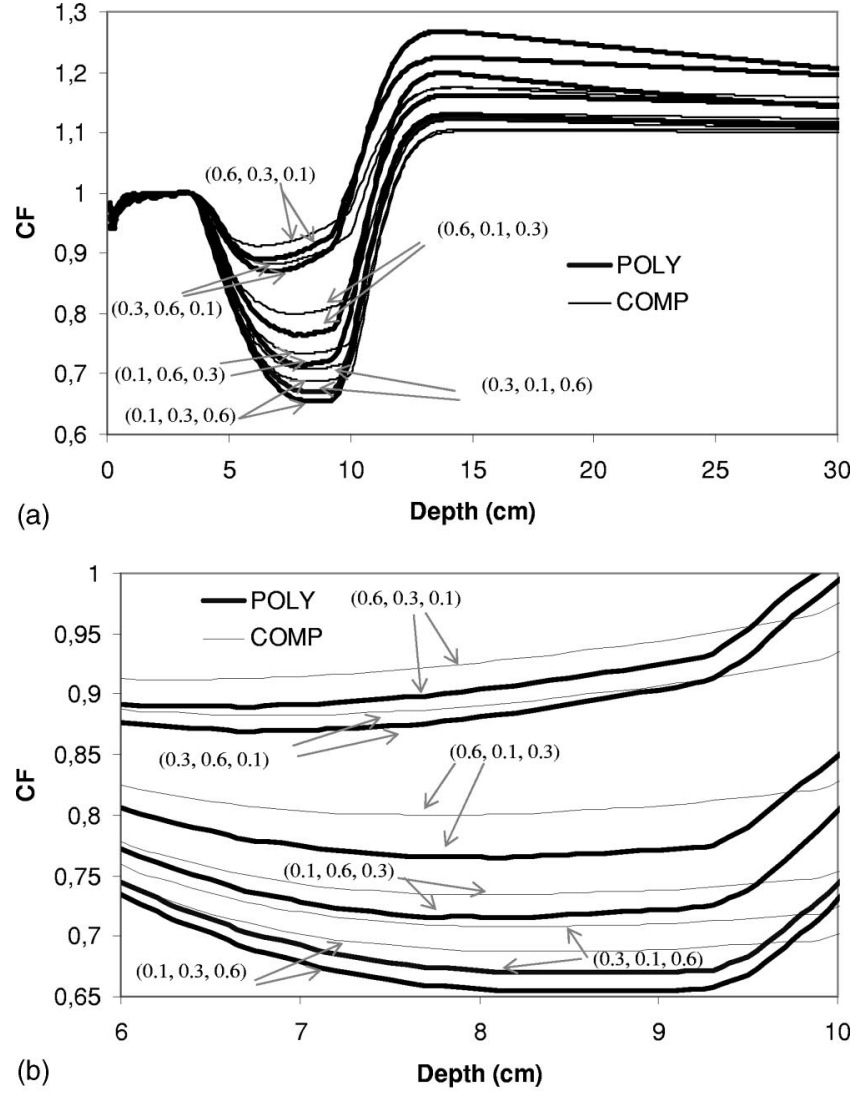

FIG. 8. The lung correction factors or CF curves for the six different spectra are shown for a field size of $3 \times 3 \mathrm{~cm}^{2}$. The polyenergetic method of the convolution algorithm is compared to the component implementation (same curves from Fig. 6). In (b), a closer look at the curves inside the lung slab is provided.

separation will be regarded as being superior with greater resolving capability. This advantage might be compromised depending on the geometry/irradiation conditions (e.g., field size). In this section the relative strengths of the tests are compared.

Table II displays the ratios of the various separations $S(i, j)$ for the CF test relative to the $D_{20} / D_{10}$ test for all field sizes. The ratios for the smallest field, $3 \times 3 \mathrm{~cm}^{2}$, are all greater than unity. The improvement for this field is at least a factor of 1.5. This advantage of the CF test over the $D_{20} / D_{10}$ test is diminished as the field size is increased. This is expected since the perturbation caused by a lung slab in a water phantom is diminished for larger fields as the electron disequilibrium is reduced.

In Table III, the ratios of the different separations $S(i, j)$ for the $\mathrm{LD}_{\text {build-up }}$ test relative to the $D_{20} / D_{10}$ test are shown for all field sizes. The advantage of the $\mathrm{LD}_{\text {build-up }}$ test over the $D_{20} / D_{10}$ test is dominant for all field sizes with the ratios often near 10 .

The last comparison is for the $\mathrm{NA}_{\text {build-up }}$ test relative to the $D_{20} / D_{10}$ test. The ratios of the $S(i, j)$ are displayed in Table IV. Similar conclusion can be drawn as for the $\mathrm{LD}_{\text {build-up }}$ (Table III). That is, the two buildup tests $\mathrm{NA}_{\text {build-up }}$ and $\mathrm{LD}_{\text {build-up }}$ with different handling of normalization are each superior to the $D_{20} / D_{10}$ test. However, the magnitude of this superiority differs.

\section{B. Experimental data}

\section{Practical example}

A fit between the convolution/superposition and the measurements was obtained by means of the CF test. Figure 9 shows the agreement between the calculation with the twobin $15 \mathrm{MV}$ spectrum and the measurements for a $3 \times 3 \mathrm{~cm}^{2}$ field $\mathrm{CF}$ experiment. The input spectrum for the convolution code used the 2 and $10 \mathrm{MeV}$ energy bins weighed to 0.733 and 0.267 , respectively. Figure 10 shows the depth doses in water for all four field sizes $\left(3 \times 3 \mathrm{~cm}^{2}, 5 \times 5 \mathrm{~cm}^{2}, 10\right.$ $\times 10 \mathrm{~cm}^{2}$, and $20 \times 20 \mathrm{~cm}^{2}$ ). At this point, the spectrum being selected, one can choose as we did to normalize the data at a depth of $10 \mathrm{~cm}$. The agreement between calculated and experimental data is within $2 \%$ for all curves beyond the normalization point. Recall that the convolution calculations are seen without electron contamination added. The difference between the experimental data, which naturally include such contamination, and the calculated depth doses yields the largest discrepancy near the build-up region. The measured dose within the first centimeter depth contains larger uncertainties and potentially accounts for the differences. More precise measurements of the build-up region would require extrapolation chamber measurements and is part of work in progress. ${ }^{33}$ The behavior of the electron contamination beyond the first centimeter for field sizes $5 \times 5 \mathrm{~cm}^{2}, 10$ $\times 10 \mathrm{~cm}^{2}$, and $20 \times 20 \mathrm{~cm}^{2}$ is otherwise somewhat similar to descriptions made for the same field sizes but different energies by other authors. ${ }^{5,17}$ This difference between the experimental data and the calculated data can in principle be fit with as many parameters as needed. ${ }^{5}$ In our case, a thirdorder polynomial would do the descending part of this build-up correction. Note that this has, however, no physical meaning. Further work is required to do a proper mapping of electron contamination of physical data to some function. The depth doses in inhomogeneous lung/water phantom for $3 \times 3 \mathrm{~cm}^{2}$ and $10 \times 10 \mathrm{~cm}^{2}$ fields are shown in Fig. 11. The agreement between the calculation and the measured data is generally good, within $2 \%$, except again for the build-up region which worsens for the larger field.

\section{DISCUSSION}

\section{A. Simulation}

Both CF and $\mathrm{LD}_{\text {build-up }}$ were superior to the $D_{20} / D_{10}$ test. A detailed look at Tables II and III shows that when the lowest energy of a pair of spectra was weighted very differently, the advantage of these tests was even greater. That is to say that these tests are sensitive to the low-energy components of the spectrum, while the $D_{20} / D_{10}$ test (Table I) was mostly sensitive to the higher energy components. These observations are expected. It has been pointed out that the beam quality index $\mathrm{TPR}_{20} / \mathrm{TPR}_{10}$ might be insensitive to spectral changes in the range of 15 to $25 \mathrm{MV},{ }^{10}$ in agreement with our findings of the lower resolving ability of the $D_{20} / D_{10}$ test. 
TABLE II. Ratios of separations from $\mathrm{CF}$ and $D_{20} / D_{10}$ tests, $S_{\mathrm{CF}}(i, j) / S_{D 20 / 10}(i, j)$.

\begin{tabular}{|c|c|c|c|c|c|c|}
\hline $3 \times 3 \mathrm{~cm}^{2}$ & 1 & 2 & 3 & 4 & 5 & 6 \\
\hline 1 & & 3.3 & 2.3 & 1.5 & 1.9 & 1.8 \\
\hline 2 & & & 2.5 & 1.9 & 2.0 & 1.9 \\
\hline 3 & & & & 3.1 & 1.7 & 1.4 \\
\hline 4 & & & & & 2.1 & 1.9 \\
\hline 5 & & & & & & 4.6 \\
\hline \multicolumn{7}{|l|}{6} \\
\hline $5 \times 5 \mathrm{~cm}^{2}$ & 1 & 2 & 3 & 4 & 5 & 6 \\
\hline 1 & & 2.7 & 1.9 & 1.1 & 1.6 & 1.4 \\
\hline 2 & & & 2.0 & 1.4 & 1.6 & 1.5 \\
\hline 3 & & & & 2.7 & 1.4 & 1.1 \\
\hline 4 & & & & & 1.7 & 1.5 \\
\hline 5 & & & & & & 5.5 \\
\hline \multicolumn{7}{|l|}{6} \\
\hline $10 \times 10 \mathrm{~cm}^{2}$ & 1 & 2 & 3 & 4 & 5 & 6 \\
\hline 1 & & 0.5 & 0.4 & 0.5 & 0.4 & 0.4 \\
\hline 2 & & & 0.4 & 0.4 & 0.4 & 0.4 \\
\hline 3 & & & & 0.6 & 0.4 & 0.3 \\
\hline 4 & & & & & 0.4 & 0.4 \\
\hline 5 & & & & & & 2.8 \\
\hline \multicolumn{7}{|l|}{6} \\
\hline $20 \times 20 \mathrm{~cm}^{2}$ & 1 & 2 & 3 & 4 & 5 & 6 \\
\hline 1 & & 0.2 & 0.1 & 0.1 & 0.1 & 0.1 \\
\hline 2 & & & 0.1 & 0.1 & 0.1 & 0.1 \\
\hline 3 & & & & 0.6 & 0.1 & 0.1 \\
\hline 4 & & & & & 0.1 & 0.1 \\
\hline 5 & & & & & & 0.4 \\
\hline 6 & & & & & & \\
\hline
\end{tabular}

The determination of high-energy components is difficult since the mass attenuation coefficient is slowly varying with energy. ${ }^{15}$ The rapid falloff with depth of the low energies will certainly influence the build-up region but is less likely for depths of 20 even $10 \mathrm{~cm}$. The high energies were not expected to influence the CF test so much since the attenuation of high energies beam by a lung slab is less remarkable. A similar experiment, for which the results are not shown, was repeated in which we used the ratios of dose at depths of 4 and $10 \mathrm{~cm}$ in water $\left(D_{10} / D_{4}\right.$ test). These depths correspond to the lung emplacement in the CF test. Only to say, the $D_{10} / D_{4}$ test and the $D_{20} / D_{10}$ test had the same discriminating power with ratios of separation of the two tests neighboring unity.

The density scaling ${ }^{34}$ for accounting for density changes is known to have some limitations for low densities ${ }^{35}$ and does not apply to high- $Z$ material. Superposition models have been known to overestimate dose in layer beyond a high-to-low density interface and underestimate dose following a low-to-high interface. ${ }^{35,36}$ Attempts have been made to incorporate electron transport ${ }^{37,38}$ and more specifically in the context of the convolution algorithm. ${ }^{36,39}$ This implies that it is not clear whether the discrepancies observed were due to an inappropriate spectrum or to the scaling theorem.

The $\mathrm{LD}_{\text {build-up }}$ test relies on interface effects. The interface here is the leading edge of the media. Due to this fact, there are edge effects which need to be carefully taken into account-namely electron contamination. We have not addressed this issue at all in this paper. As well, our calculations of the $\mathrm{LD}_{\text {build-up }}$ test depended heavily on the accuracy of our interpolation of the discrete dose data and the computation of derivatives of these interpolated functions. Each of these numerical processes introduces noise into the computation which needs to be acknowledged. While such noise has not had a large effect on our results, minimizing these technical problems will lead to greater efficacy of the $\mathrm{LD}_{\text {build-up }}$ test. Because of these issues, we believe that the $\mathrm{CF}$ test may well be the superior test for discriminating spectra in small fields.

The CF test depends on interface effects in an obvious way. The CF test does not work so well for large fields due to the fact that electronic equilibrium is re-established. In fact, it was inferior to the $D_{20} / D_{10}$ test for the two largest fields considered. The electron contamination can be neglected in the build-up region of small fields, which makes the CF test even more attractive. Bloch et al. ${ }^{15}$ have exploited measurements in the build-up region of small fields exempt of electron contamination, complementary to transmission measurements, to determine the high-energy components of a spectrum.

The CF test appears to also give useful information for spectra in the polyenergetic approximation of the convolution/superposition code. Despite the fact that the polyenergetic approximation occasionally leads to "similar" depth doses in water to the ones generated with the component method, it is not sufficient to guarantee further closeness of the two methods. The CF ratios for a given spectrum were even further apart for the two methods. A full comparison of the two implementations is, however, beyond the scope of this paper. It can, however, be said that other than the lengthier process, the component method can be thought of as advantageous for simplifying spectrum fitting. In the polyenergetic implementation, iterative methods to fit the data are often used to adjust the weights for the terma. The kernel, however, is not necessarily adjusted to the same weighting. This mismatch has no physical meaning. Further advantage of the component method is that the convolution/ superposition can be better understood and ameliorated by potentially studying individually the behavior of each energy against Monte Carlo simulations.

The $\mathrm{NA}_{\text {build-up }}$ test, while superior to the $D_{20} / D_{10}$ test for all field sizes, suffers from ambiguities due to the fact that it depends explicitly on a choice of normalization. In our study we have normalized each depth dose curve according to the area under that particular curve. As this area has the interpretation of total dose, we have normalized the depth dose from a given spectrum according to its total energy content. We have chosen the particular normalization as we expect it to be directly related to the total energy deposited in the media and is hence an externally controllable parameter which can be fixed without reference to the details of the target media. Remarkably, fixing the constant total dose does not appear to guarantee the conservation of deposited energy on the central axis. The area under the depth dose curve in water does not 
TABLE III. Ratios of separations $\mathrm{LD}_{\text {build-up }}$ to $D_{20} / D_{10}$ tests, $S_{\mathrm{LD}}(i, j) / S_{D 20 / 10}(i, j)$.

\begin{tabular}{|c|c|c|c|c|c|c|}
\hline $3 \times 3 \mathrm{~cm}^{2}$ & 1 & 2 & 3 & 4 & 5 & 6 \\
\hline 1 & & 10.1 & 8.8 & 6.0 & 8.9 & 7.3 \\
\hline 2 & & & 9.0 & 7.0 & 8.6 & 7.5 \\
\hline 3 & & & & 11.6 & 9.9 & 7.2 \\
\hline 4 & & & & & 9.5 & 8.6 \\
\hline 5 & & & & & & 40.0 \\
\hline \multicolumn{7}{|l|}{6} \\
\hline $5 \times 5 \mathrm{~cm}^{2}$ & 1 & 2 & 3 & 4 & 5 & 6 \\
\hline 1 & & 10.5 & 9.3 & 5.1 & 9.3 & 7.6 \\
\hline 2 & & & 9.4 & 6.6 & 9.4 & 8.0 \\
\hline 3 & & & & 13.4 & 10.6 & 7.4 \\
\hline 4 & & & & & 10.8 & 8.9 \\
\hline 5 & & & & & & 57.6 \\
\hline \multicolumn{7}{|l|}{6} \\
\hline $10 \times 10 \mathrm{~cm}^{2}$ & 1 & 2 & 3 & 4 & 5 & 6 \\
\hline 1 & & 12.7 & 9.2 & 4.4 & 9.5 & 7.4 \\
\hline 2 & & & 9.4 & 5.7 & 9.4 & 7.6 \\
\hline 3 & & & & 18.0 & 10.5 & 6.8 \\
\hline 4 & & & & & 11.4 & 8.6 \\
\hline 5 & & & & & & 319.0 \\
\hline \multicolumn{7}{|l|}{6} \\
\hline $20 \times 20 \mathrm{~cm}^{2}$ & 1 & 2 & 3 & 4 & 5 & 6 \\
\hline 1 & & 25.2 & 10.7 & 3.8 & 11.2 & 8.0 \\
\hline 2 & & & 11.8 & 5.3 & 11.6 & 8.3 \\
\hline 3 & & & & 59.8 & 12.5 & 10.8 \\
\hline 4 & & & & & 15.3 & 9.9 \\
\hline 5 & & & & & & 27.1 \\
\hline 6 & & & & & & \\
\hline
\end{tabular}

agree with the area under the curve of an inhomogeneous water/lung depth dose when a beam with the same spectrum is used in each medium. This means that a same unit flux of one spectrum does not provide the same total dose deposition on the central axis in water as in water/lung media.

Unfortunately, the criteria of nonambiguity are not met for some popular choices of normalization. This can be seen in the following thought experiment involving a commonly used normalization choice in a situation which is not clinically relevant. It is common practice to force beam normalization at a certain depth, e.g., either $d_{\max }$ or $10 \mathrm{~cm}$ depth. Such a choice makes it difficult to compare physically distinct situations where we expect dose at depth of $10 \mathrm{~cm}$ to differ. Taking this protocol to an extreme, in order to match depth doses for a $10 \mathrm{MeV}$ beam to that of a $200 \mathrm{keV}$ beam at depth of $10 \mathrm{~cm}$, we would require an exponentially large/ small normalization factor. This is not a comfortable situation and it demonstrates that fixing normalization at a particular depth is not a benign process but rather imposes outside bias on the physics of the situation. From a more practical point of view, we can see direct qualitative effects of this standard normalization in the data presented in Fig. 5, where depth doses are normalized with respect to total dose. Here, we see that there is no single point where all dose curves intersect, and by forcing them to do so leads to arti- ficial amplification of doses in either the build-up region or at depth.

As there are ambiguities associated with the choice of normalization, we propose a procedure for avoiding the possibilities of biasing analyses. In this paper we have presented a number of normalization-independent tests in order to measure depth dose curves. These tests and other normalizationindependent tests have the capability to supply all information regarding energy spectra which is relevant to the calculation of depth doses via the convolution/superposition component algorithm. This is due to the fundamental fact that the algorithm is linear and all results produced by it are equivalent up to normalization. Consequently, we can calculate doses or energy spectra without regard to overall normalization and only worry about fixing a definite normalization as a final step of the calculation, since it is practical to do so for calibration purposes.

\section{B. Practical example}

We have shown the results of our fit to actual $15 \mathrm{MV}$ physical data to illustrate the potential for the spectrum discrimination methodology proposed. In our selection of energy bins and weights, we required that the small field $\mathrm{CF}$ calculated data, exempt from electron contamination, agreed with the corresponding measured data. We obtained a spectrum that would fit both water and inhomogeneous lung depth doses for the small field. As the field size increase, electron contamination is expected and a correction for this effect must be added. In principle, there should also be an account for the spectral change away from the central axis. All these behaviors must be properly modeled and accounted for in the convolution/superposition algorithm, but they are not perfectly modeled for the work described in this paper. A more suitable approach for improving the code is to follow a methodology which limits the amount of arbitrariness and inconsistency, e.g., arbitrary normalization of depth doses patched with electron contamination ${ }^{5}$ and kernel inconsistent with the spectrum of the terma. The $3 \times 3 \mathrm{~cm}^{2}$ field experimental data mean measurements in conditions where lateral electronic equilibrium might not be existent. The issue of accurate measurement interpretation in those circumstances will not be addressed here.

We have only considered two components for the spectrum (and three for the simulation). This is an arbitrary choice. We expect our results to hold analogously for spectra composed of several energies. In all cases, the observation that the depth dose for some single energy cannot be obtained by adding depth doses from other energies directly guarantees that different spectra (unique up to normalization) produce different $\mathrm{CF}$ curves.

\section{CONCLUSION}

We have presented guidelines to ease the spectrum determination for the component-based convolution/superposition dose calculation algorithm by making use of interface ef- 
TABLE IV. Ratios of separations $\mathrm{NA}_{\text {build-up }}$ to $D_{20} / D_{10}$ tests, $S_{N A}(i, j) / S_{D 20 / 10}(i, j)$.

\begin{tabular}{|c|c|c|c|c|c|c|}
\hline $3 \times 3 \mathrm{~cm}^{2}$ & 1 & 2 & 3 & 4 & 5 & 6 \\
\hline 1 & & 3.7 & 4.0 & 17.0 & 3.7 & 4.9 \\
\hline 2 & & & 3.8 & 9.2 & 3.3 & 4.3 \\
\hline 3 & & & & 2.8 & 4.1 & 6.9 \\
\hline 4 & & & & & 2.9 & 3.3 \\
\hline 5 & & & & & & 4.9 \\
\hline \multicolumn{7}{|l|}{6} \\
\hline $5 \times 5 \mathrm{~cm}^{2}$ & 1 & 2 & 3 & 4 & 5 & 6 \\
\hline 1 & & 5.9 & 6.0 & 25.7 & 5.3 & 7.0 \\
\hline 2 & & & 5.8 & 14.1 & 4.7 & 6.2 \\
\hline 3 & & & & 0.0 & 5.6 & 9.5 \\
\hline 4 & & & & & 4.2 & 4.7 \\
\hline 5 & & & & & & 6.1 \\
\hline \multicolumn{7}{|l|}{6} \\
\hline $10 \times 10 \mathrm{~cm}^{2}$ & 1 & 2 & 3 & 4 & 5 & 6 \\
\hline 1 & & 38.0 & 27.9 & 52.8 & 22.6 & 26.2 \\
\hline 2 & & & 28.2 & 49.3 & 20.6 & 24.5 \\
\hline 3 & & & & 29.2 & 22.2 & 29.7 \\
\hline 4 & & & & & 19.4 & 19.4 \\
\hline 5 & & & & & & 69.6 \\
\hline \multicolumn{7}{|l|}{6} \\
\hline $20 \times 20 \mathrm{~cm}^{2}$ & 1 & 2 & 3 & 4 & 5 & 6 \\
\hline 1 & & 215.1 & 149.5 & 321.9 & 114.7 & 104.6 \\
\hline 2 & & & 170.2 & 152.7 & 110.5 & 103.2 \\
\hline 3 & & & & 84.7 & 110.0 & 90.6 \\
\hline 4 & & & & & 120.9 & 87.3 \\
\hline 5 & & & & & & 51.9 \\
\hline 6 & & & & & & \\
\hline
\end{tabular}

fects. While we have used these effects, we have not exhausted their utility in determining spectra for use in the convolution/superposition algorithm.

There are still several questions which must be answered,



FIG. 9. Comparison between the CF curves measured experimentally and calculated by the convolution algorithm with a two-component fitted spectrum. The data for a $15 \mathrm{MV}, 3 \times 3 \mathrm{~cm}^{2}$ photon beam are shown, and were those used for the fitting. one of which is the choice of appropriate basis of discrete spectra which capture the physics of the continuous spectrum as defined by the dose we calculate via the convolution/ superposition algorithm. In order to implement a decimation procedure we must have a method for determining the energies and relative strengths of monoenergetic beams which will serve to model the full output of a realistic linac; more work needs to be done in this area.

We are aware that the convolution/superposition algorithm might have limitations in predicting dose, e.g., build-up region and inhomogeneity. Better knowledge of the build-up region is also needed. Definitely, there are more tests that can be explored to identify spectra. As the test spectrum passes more tests, it should converge to the exact spectrum. Off-axis data were not part of this study but are also thought to offer valuable information and would be part of future work. A proper examination of off-axis data will require proper off-axis spectrum modeling of the algorithm.

\section{ACKNOWLEDGMENT}

This work has been supported in part by NIH Grant P01CA59827.

\section{APPENDIX A: CONVOLUTION/SUPERPOSITION DOSE CALCULATION}

There exist many implementations based on the convolution/superposition principle for which the sole intent is to calculate dose as accurately as possible in a reasonable amount of time. The dose from the component convolution method is calculated from the following integrals: ${ }^{1,16,29}$

$$
D(r)=\int_{0}^{E_{0}} \int_{V} \frac{\mu(E)}{\rho} \cdot E \Phi\left(r^{\prime}, E\right) \cdot A\left(r-r^{\prime}, E\right) \cdot d^{3} r^{\prime} d E,
$$

where $E$ is the energy, $A\left(r-r^{\prime}, E\right)$ is the energy deposition kernel, and $\mu / \rho$ is the linear mass attenuation coefficient. The primary photon fluence $\Phi(r, E$,$) is expressed as$

$$
\Phi\left(r^{\prime}, E\right)=\Phi\left(z_{0}, E\right) \cdot \exp \left(-\mu\left|z^{\prime}-z_{0}\right|\right) .
$$

Equation (A1) can be rewritten as

$$
D(r)=\int_{0}^{E_{0}} W(E) \cdot f(r, E) \cdot d E,
$$

where $W(E)$ is a non-negative weight function. The function $f(r, E)$ represents the dose at point $r$ due to a monoenergetic photon beam. $D(r)$ represents the total dose at $r$ after summing over the energy spectrum $W(E)$

$$
f(r, E)=\int_{V} \frac{\mu(E)}{\rho} \cdot E \Phi\left(r^{\prime}, E\right) \cdot A\left(r-r^{\prime}, E\right) \cdot d^{3} r^{\prime} .
$$

In the discrete case for numerical evaluation, Eq. (A4) becomes 


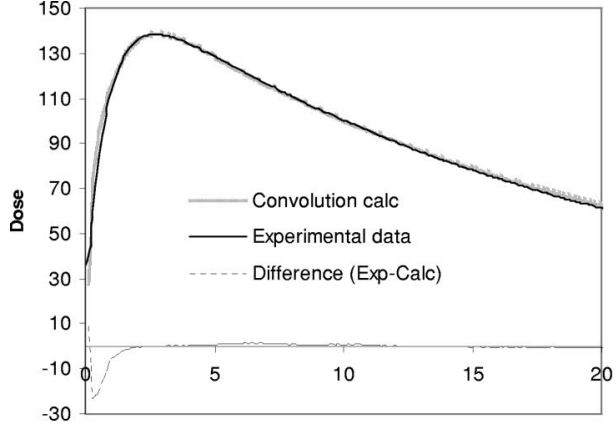

(a)

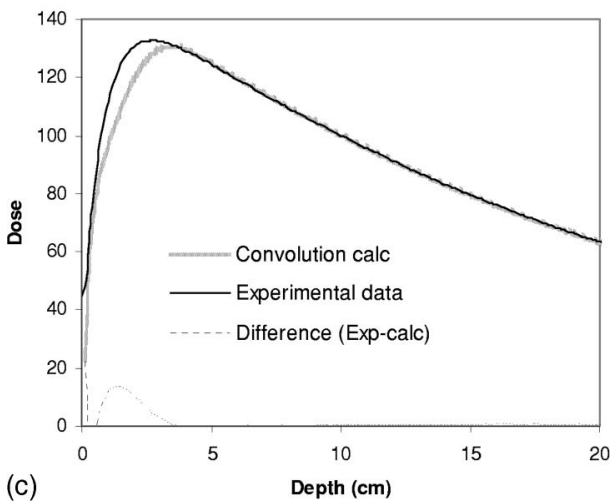

(c)

$$
F(r)=\sum_{i=1}^{n} w_{i} \cdot f\left(r, E_{i}\right) .
$$

The three-component method $(n=3)$, with only three energy bins representative of the entire beam spectrum, is intended as a faster alternative to the intuitively accurate multicomponent implementation for polyenergetic beams. An electron contamination term (EC) can be added to the dose calculation, such that Eq. (A3) becomes

$$
D(r)=\int_{0}^{E_{0}} W(E) \cdot f(r, E) \cdot d E+E C .
$$

In the polyenergetic implementation, both the kernel and the terma are preaveraged over the energies. The equation for polyenergetic approximation analogous to Eq. (A1) looks like this:

$$
\begin{aligned}
D(r)= & {\left[\int_{V} \frac{\int_{0}^{E o}(\mu / \rho) \cdot E \Phi d E}{\int_{0}^{E o} E \Phi d E}\right] \cdot\left[\frac{\int_{0}^{E o} E \Phi d E}{\int_{0}^{E o} \Phi d E}\right] } \\
& \cdot\left[\frac{\int_{0}^{E o}(\mu / \rho) \cdot E \Phi A d E}{\int_{0}^{E o}(\mu / \rho) \cdot E \Phi d E}\right] \cdot d^{3} r^{\prime} .
\end{aligned}
$$

We can define the averages of the quantities in the brackets by

$$
\begin{aligned}
\frac{\bar{\mu}}{\rho}\left(r^{\prime}\right) & =\frac{\left.\int_{0}^{E o}(\mu / \rho)(E) \cdot E \Phi * r^{\prime}, E\right) d E}{\int_{0}^{E o} E \Phi\left(r^{\prime}, E\right) d E}, \\
\bar{E}\left(r^{\prime}\right) & =\frac{\int_{0}^{E o} E \Phi\left(r^{\prime}, E\right) d E}{\int_{0}^{E o} \Phi\left(r^{\prime}, E\right) d E} \\
\bar{\Phi}\left(r^{\prime}\right) & =\int_{0}^{E o} \Phi\left(r^{\prime}, e\right) d E
\end{aligned}
$$


FIG. 11. Comparison between experimental and calculated depth doses in lung/water inhomogeneous slab phantom for (a) $3 \times 3 \mathrm{~cm}^{2}$ and (b) 10 $\times 10 \mathrm{~cm}^{2} 15 \mathrm{MV}$ photon beams. The calculations by the convolution algorithm made use of the two-component fitted spectrum. The overall units of dose are scaled arbitrarily. 
and,

$$
\bar{A}\left(\left|r^{\prime}-r\right|\right)=\frac{\int_{0}^{E o} \frac{\mu}{\rho}(E) \cdot E \Phi\left(r^{\prime}, E\right) \cdot A\left(\left|r^{\prime}-r\right|, E\right) d E}{\int_{0}^{E o} \frac{\mu}{\rho}\left(r^{\prime}\right) \cdot \bar{E}\left(r^{\prime}\right) \cdot \Phi\left(r^{\prime}, E\right) d E} .
$$

Therefore, $D(r)$ can be expressed as

$$
D(r)=\int_{V} \frac{\bar{\mu}}{\rho}\left(r^{\prime}\right) \cdot \bar{E}\left(r^{\prime}\right) \cdot \Phi\left(r^{\prime}\right) \cdot \bar{A}\left(r^{\prime},\left|r^{\prime}-r\right|\right) \cdot d^{3} r^{\prime} .
$$

\section{APPENDIX B: LUNG CORRECTION FACTOR IN PRESENCE OF ELECTRON CONTAMINATION}

Both the physical doses in lung/water and water media include an electron contamination (EC) term

$$
\mathrm{CF}=\frac{D^{\text {lung }}}{D^{\text {water }}}=\frac{D_{\text {noEC }}^{\text {lung }}+\mathrm{EC}}{D_{\text {noEC }}^{\text {water }}+\mathrm{EC}}
$$

Here, we have defined dose without electron contamination contribution $D_{\text {noEC }}$ for both inhomogeneous lung/water and water-only media. We make this distinction because we chose not to include such corrections in our calculations. One may be concerned that this omission might affect the values of the $\mathrm{CF}$ test since it definitely has an effect on measured dose. We now show that under common circumstances this is not the case. Rewriting Eq. (B1), we can define a correction factor $\mathrm{CF}_{\text {noEC }}$, which does not take into account electron contamination

$$
\mathrm{CF}=\frac{D_{\text {noEC }}^{\text {lung }}}{D_{\text {noEC }}^{\text {water }}}\left(\frac{1+\mathrm{EC} / D_{\text {noEC }}^{\text {lung }}}{1+\mathrm{EC} / D_{\text {noEC }}^{\text {water }}}\right)=\mathrm{CF}_{\text {noEC }}\left(\frac{1+\mathrm{EC} / D_{\text {noEC }}^{\text {lung }}}{1+\mathrm{EC} / D_{\text {noEC }}^{\text {water }}}\right) .
$$

The factor which determines the ratio of $\mathrm{CF}$ to $\mathrm{CF}_{\mathrm{noEC}}$ is essentially unity when either the inhomogeneity due to the lung or the electron contamination EC can be ignored. This condition is met for lung beginning at several centimeters depth as the effect of electron contamination decays exponentially.

${ }^{a)}$ Current address: Grand River Regional Cancer Center, Kitchener, Ontario N2G 1G3, Canada. Electronic mail: paule.charland@grhosp.on.ca

${ }^{1}$ T. R. Mackie, J. W. Scrimger, and J. J. Battista, "A convolution method of calculating dose for 15 MV x rays," Med. Phys. 12, 188-196 (1985).

${ }^{2}$ M. Miften, M. Wiesmeyer, S. Monthofer, and K. Krippner, "Implementation of FFT convolution and multigrid superposition models in the FOCUS RTP system,” Phys. Med. Biol. 45, 817-833 (2000).

${ }^{3}$ P. W. Hoban, D. C. Murray, P. E. Metcalfe, and W. H. Round, "Superposition dose calculation in lung for $10 \mathrm{MV}$ photons," Australas. Phys. Eng. Sci. Med. 13, 81-92 (1990).

${ }^{4}$ A. Ahnesjo, P. Andreo, and A. Brahme, "Calculation and application of point spread functions for treatment planning with high energy photon beams," Acta Oncol. 26, 49-56 (1987).

${ }^{5}$ G. Starkschall, R. E. Steadham, Jr., R. A. Popple, S. Ahmad, and I. Rosen, "Beam-commissioning methodology for a three-dimensional convolution/superposition photon dose algorithm," J. Appl. Clin. Med. Phys. 1, 8-27 (2000).
${ }^{6}$ J. Brownridge, S. Samnick, P. Tipton, J. Veselka, and N. Yeh, "Determination of the photon spectrum of a clinical accelerator," Med. Phys. 11, 794-797 (1984).

${ }^{7}$ B. A. Faddegon, C. K. Ross, and D. W. O. Rogers, "Forward-directed bremsstrahlung of 10 to $30 \mathrm{MeV}$ electrons incident on thick targets of $\mathrm{Al}$ and Pb," Med. Phys. 17, 773-785 (1990).

${ }^{8}$ D. J. Landry and D. W. Andersen, "Measurements of accelerator bremsstrahlung spectra with a high efficiency Ge detector," Med. Phys. 18, 527-532 (1991).

${ }^{9}$ W. H. Hinson and J. D. Bourland, "Spectral reconstruction of high energy photon beams for kernel based dose calculations," Med. Phys. 29, 1789-1796 (2002)

${ }^{10}$ A. Nisbet, H. Weatherburn, J. D. Fenwich, and G. McVey, "Spectral reconstruction of clinical megavoltage photon beams and implications of spectral determination on dosimetry of such beams," Phys. Med. Biol. 43, 1507-1521 (1998).

${ }^{11}$ P. Francois, F. Coste, J. Bonnet, and O. Caselles, "Validation of reconstructed bremsstrahlung spectra between $6 \mathrm{MV}$ and $25 \mathrm{MV}$ from measured transmission data," Med. Phys. 24, 769-773 (1997).

${ }^{12}$ A. Catala, P. Francois, J. Bonnet, and Ch. Scouarnec, "Reconstruction of 12 MV bremsstrahlung spectra from measured transmission data by direct resolution of the numeric system $\mathrm{AF}=\mathrm{T}$," Med. Phys. 22, 3-10 (1995).

${ }^{13}$ P. H. Huang, K. R. Kase, and B. E. Bjärngard, "Simulation studies of 4-MV x-ray spectral reconstruction by numerical analysis of transmission data," Med. Phys. 9, 695-702 (1981).

${ }^{14}$ B. R. Archer, P. R. Almond, and L. K. Wagner, "Application of a Laplace transform pair model for high x-ray spectral reconstruction," Med. Phys. 12, 630-633 (1985).

${ }^{15} \mathrm{P}$. Bloch and J. McDonough, "Extraction of the photon spectra from measured beam parameters," Med. Phys. 25, 752-757 (1998).

${ }^{16}$ N. Papanikolaou, R. T. Mackie, C. Meger-Wells, M. Gehring, and P. Reckwerdt, "Investigation of the convolution method for polyenergetic spectra," Med. Phys. 20, 1327-1336 (1993).

${ }^{17} \mathrm{~A}$. Ahnesjö and P. Andreo, "Determination of the effective bremsstrahlung spectra and electron contamination for photon dose calculations," Phys. Med. Biol. 34, 1451-1464 (1989).

${ }^{18}$ P. Bloch, M. D. Altschuler, B. F. Bjarngard, A. Kassaee, and J. McDonough, "Determining clinical photon beam spectra from measured depth dose with the Cimmino algorithm," Phys. Med. Biol. 45, 171-183 (2000).

${ }^{19}$ R. Mohan, C. Chui, and L. Lidofsky, "Energy and angular distributions of photons from medical linear accelerators," Med. Phys. 12, 592-597 (1985).

${ }^{20}$ E. L. Chaney, T. J. Cullip, and J. A. Gabriel, "A Monte Carlo study of accelerator head scatter," Med. Phys. 21, 1383-1390 (1994).

${ }^{21}$ D. W. O. Rogers, B. A. Faddegon, G. X. Ding, C.-M. Ma, J. We, and T. R. Mackie, "BEAM: A Monte Carlo to simulate radiotherapy treatment units," Med. Phys. 22, 503-524 (1995).

${ }^{22}$ D. Sheikh-Bagheri and D. W. O. Rogers, "Monte Carlo calculation of nine megavoltage photon beam spectra using the BEAM code," Med. Phys. 29, 391-402 (2002).

${ }^{23}$ D. Sheikh-Bagheri and D. W. O. Rogers, "Sensitivity of megavoltage photon beam Monte Carlo simulations to electron beam and other parameters," Med. Phys. 29, 379-390 (2002).

${ }^{24}$ J. M. Lydon, "Photon dose calculations in homogeneous media for a treatment planning system using a collapsed cone superposition convolution algorithm," Phys. Med. Biol. 43, 1813-1822 (1998).

${ }^{25}$ D. S. Followill, R. C. Tailor, V. M. Tello, and W. F. Hansen, "An empirical relationship for determining photon beam quality in TG-21 from a ratio of percent depth doses," Med. Phys. 25, 1202-1205 (1998).

${ }^{26}$ B. A. Fraass, K. Doppke, M. A. Hunt, G. J. Kutcher, G. Starkschall, R. Stern, and J. Van Dyk, "American Association of Physicists in Medicine Radiation Therapy Committee Task Group 53: Quality assurance for clinical radiotherapy treatment planning," Med. Phys. 25, 1773-1829 (1998).

${ }^{27}$ K. A. Gifford, D. S. Followill, and H. H. Liu, "Verification of the accuracy of a photon dose-calculation algorithm," J. Appl. Clin. Med. Phys. 3, 26-45 (2002).

${ }^{28} \mathrm{~J}$. L. Bedford, "Speed versus accuracy in a fast convolution photon dose calculation for conformal radiotherapy," Phys. Med. Biol. 47, 3475-3484 (2002).

${ }^{29}$ H. H. Liu, T. R. Mackie, and E. C. McCullough, "Correcting kernel 
tilting and hardening in convolution/superposition dose calculations for clinical divergent and polychromatic photon beams," Med. Phys. 24, 1729-1741 (1997).

${ }^{30}$ American Association of Physicists in Medicine Radiation Therapy Committee Task Group 21, "A protocol for determination of absorbed dose from high-energy photon and electron beams," Med. Phys. 10, 741-771 (1983).

${ }^{31}$ R. Rice, J. L. Hansen, L. M. Chin, B. J. Mijnheer, and B. E. Bjärngard, "The influence of ionization chamber and phantom design on the measurement of lung dose in photon beams," Med. Phys. 15, 884-890 (1988).

${ }^{32}$ R. Jeraj, G. Olivera, P. Reckwerdt, J. Smilowitz, and T. Mackie, "Supported commissioning of non-Monte Carlo based treatment planning systems," Med. Phys. 29, 1289 (Abstract) (2002).

${ }^{33}$ S. Yokoyama, P. Roberson, J. Moran, D. Litzenberg, and B. Fraass, "Build-up region dependence on photon dose delivery technique for IMRT,” Med. Phys. 29, 1315 (Abstract) (2002).
${ }^{34}$ J. E. O'Connor, “The density scaling theorem applied to lateral electronic equilibrium," Med. Phys. 11, 678-680 (1984).

${ }^{35}$ M. K. Woo and J. R. Cunningham, "The validity of the density scaling method in primary electron transport for photon and electron beams," Med. Phys. 17, 187-194 (1990).

${ }^{36} \mathrm{P}$. J. Keall and P. Hoban, "Accounting for primary electron scatter in X-ray beam convolution,” Med. Phys. 22, 1413-1418 (1995).

${ }^{37}$ C. X. Yu, T. R. Mackie, and J. W. Wong, "Photon dose calculation incorporating explicit electron transport," Med. Phys. 22, 1157-1165 (1995).

${ }^{38}$ P. L. Petti, R. K. Rice, B. J. Mijnheer, L. M. Chin, and B. E. Bjarngard, "An inhomogeneity model for photon beams incorporating electron transport," Med. Phys. 14, 349-354 (1987).

${ }^{39}$ O. A. Sauer, "Calculation of dose distributions in the vicinity of high- $Z$ interfaces for photon beams," Med. Phys. 22, 1685-1690 (1995). 